\title{
The study on free vibration of elastically restrained beams carrying various types of attachments with arbitrary distributions
}

\author{
Heye Xiao ${ }^{\mathrm{a}, *}$, Meiping sheng ${ }^{\mathrm{a}}$, Zhihong $\mathrm{Liu}^{\mathrm{a}}$ and Zhaoyu Wei ${ }^{\mathrm{b}}$ \\ ${ }^{a}$ College of Marine, Northwestern Polytechnical University, Xi'an, Shaanxi, China \\ ${ }^{\mathrm{b}}$ School of Naval Architecture and Ocean Engineering, Jiangsu University of Science and Technology, Zhenjiang, \\ Jiangsu, China
}

Received 24 March 2012

Revised 4 October 2012

Accepted 19 October 2012

\begin{abstract}
The flexible beams carrying attachments often appear in engineering structures, modal analysis of those structures is important and necessary in structural design. This manuscript develops a proposed analytical method as a general tool for solving the free vibration of varying cross-section beams carrying various types of attachments with different distributions and arbitrary boundary conditions. In current practice, the natural frequencies of beam carrying lumped and uniform sprung mass and resting on Pasternak soil are calculated and compared with those in references to verify the methodology firstly. Then, the natural frequencies of beam carrying non-uniform spring-mass systems and stepped beam on Pasternak soil are calculated by the proposed method to study free vibration of beam carrying attachments with non-uniform cross section or distribution. Finally, some important conclusions are derived from results, which reveal that distribution density of spring-mass system at peaks of the mode shape makes dominate effects on the change of the natural frequencies at that mode and the natural frequencies of the stepped beam resting on elastic foundation are more sensitive to the increase of soil parameters.
\end{abstract}

Keywords: Natural frequency, Euler-Bernoulli beam, distributed attachment

\section{Introduction}

In the fields of mechanical, civil and aeronautical engineering, the flexural beam carrying attachments with different types, such as the mass, motors and oscillators, could be widely observed. In the past years, extensive researches have been carried out to analyze the free vibration of these kinds of beams with general boundary conditions, which aims to avoid harmful vibrations in these structures. In these studies, the beams are considered carrying different masses, stiffness and other attachment styles at arbitrary positions.

Low [1] studied the natural frequency of an Euler-Bernoulli beam carrying a concentrated mass at an arbitrary location. In his work, the result for the fixed-fixed cases is compared with those obtained experimentally. Later, Chang [2] calculated the natural frequencies of a Bernoulli-Euler beam with Laplace transfer method, which carries arbitrarily located point masses, translational springs and viscous dampers. Further, Lin and Tsai [3] determined the natural frequencies and mode shapes of Euler-Bernoulli multi-span beam carrying multiple spring-mass systems.

${ }^{*}$ Corresponding author: Heye Xiao, College of Marine, Northwestern Polytechnical University, Xi'an, Shaanxi, China. Tel.: +86 1389284 7098; Fax: +86 0298847 4094; E-mail: raulwavell@163.com. 


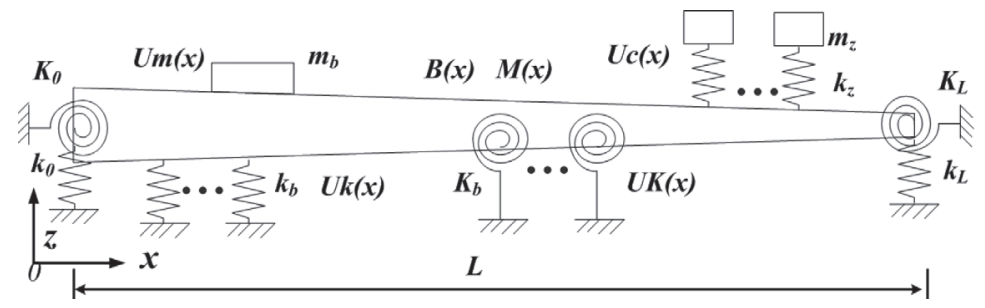

Fig. 1. Vibrating mechanical system of varying cross-section beam with attachments.

Chen [4] solved the problem of free vibration of arbitrarily thick beams resting on a Pasternak elastic foundation, within his research that the state space method combining with the differential quadrature method are applied. Other studies of the influences of variable attachments on slender beam vibrations are given in Refs. [5-17].

As to the researches on the beam carrying attachments, the studies on beam carrying spring-mass systems have received wide attentions [3,5-9]. Gürgöze [5] considered a cantilever Euler-Bernoulli beam with several springmass systems. In his work, two alternative formulations for the frequency equation were presented. Wu and Hsu [6] used the finite element method to study the dynamic behaviors of a beam with a number of spring-mass systems. Former researchers almost focused on the free vibration of beams carrying lumped and uniform spring-mass system, while the study about the natural frequencies of the beams carrying spring-mass system with arbitrary distribution is rarely reviewed. As the beams carrying various spring-mass systems could be found their wide applications in real engineering structures, the analysis of free vibration of these structures are important and necessary.

But different methods were used to solve natural frequencies of beam carrying different types of attachments and only two articles $[18,19]$ which give details of approach to analyze free vibration of beam with lumped attachments universally are found, the development of the general method for solving natural frequencies of the beam carrying spring-mass system with different distributions is necessary preparation for analysis of free vibration of those structures and become an important part of this article.

In this paper, an approach based on the research by $\mathrm{Li}[20,21]$ is introduced to analyze the free vibration of elastically restrained beams carrying various types of attachments with arbitrary spatial distributions firstly. Some numerical examples are shown together with the solutions described in references to validate the methodology. Then, the proposed method is used to solve the natural frequencies of beam carrying spring-mass systems with five different distributions. The principle for the effects of spring-mass system with various distributions on changing its natural frequencies is also obtained. Finally, the natural frequencies of the beams resting on Pasternak soil are investigated with this paper method.

\section{Theory}

The problem of varying cross-section beams carrying various types of attachments with arbitrary spatial distributions is shown in Fig. 1. This model is of particular model to the engineers in view of their rather frequent usage in actual project, especially in the case of free vibration of Euler beam resting on Pasternak soil. Thus, their study on the natural frequencies of the model is meaningful for the diagnosis of pipes in solid and dynamic design of bridges with human interactions.

In Fig. 1, the whole system is composed of Bernoulli-Euler beam and distributed attachments. One part is the Bernoulli-Euler beam with varying cross-section, its bending stiffness and mass are considered as bending stiffness function $B(x)$ and mass function $M(x)$ respectively. The other part is the distributed attachments; they include linear springs $k_{b}$ and rotational springs $K_{b}$, masses $m_{b}$ and a spring-mass system which consists of a spring of stiffness $k_{z}$ and a mass $m_{z}$. Both ends of beam are supported by linear springs and rotation springs, which are $k_{0}, K_{0}$ at $x=0$ and $k_{L}, K_{L}$ at $x=L$, to simulate different boundary conditions. It can be expressed as

$$
\left\{\begin{array}{ll}
\left.\overline{k_{0}} W\right|_{x=0} & =-\left.W^{\prime \prime \prime}\right|_{x=0} \\
\left.\overline{K_{0}} W^{\prime}\right|_{x=0} & =\left.W^{\prime \prime}\right|_{x=0}
\end{array},\left\{\begin{array}{ll}
\left.\overline{k_{l}} W\right|_{x=L} & =\left.W^{\prime \prime \prime}\right|_{x=L} \\
\left.\overline{K_{l}} W^{\prime}\right|_{x=L} & =-\left.W^{\prime \prime}\right|_{x=L}
\end{array},\left(\left\{\begin{array}{ll}
\overline{k_{0}} & =\frac{k_{0}}{E I} \\
\overline{K_{0}} & =\frac{K_{0}}{E I}
\end{array},\left\{\begin{array}{ll}
\overline{k_{L}} & =\frac{k_{L}}{E I} \\
\overline{K_{L}} & =\frac{K_{L}}{E I}
\end{array}\right) .\right.\right.\right.\right.
$$


In Eq. (1), the familiar homogeneous boundary conditions, such as the simply supported, clamped, and the free boundary condition, can be directly obtained by accordingly setting the spring stiffness to be an extremely large or small number.

In Fig. 1, the effects of rotational and tensional stiffness on beam are considered as their stiffness multiplying the rotational angle and displacement at their positions respectively. The mass is regarded as an inertial force on beam. Therefore, the governing differential equation for the free vibration of the beam can be written as

$$
\begin{aligned}
& \frac{\partial^{2}}{\partial x^{2}}\left(B(x) \frac{\partial^{4} W(x)}{\partial x^{4}}\right)-M(x) \omega^{2} W(x) \\
& \quad+\left(\sum_{i=1}^{N k} U k_{i}(x) k_{b, i}+\sum_{i=1}^{N c} U c_{i}(x) k_{e, i}-\omega^{2} \sum_{i=1}^{N m} U m_{i}(x) m_{b, i}\right) W(x), \\
& \quad-\sum_{i=1}^{N K} K_{i} \frac{\partial U K_{i}(x)}{\partial x} \frac{\partial W(x)}{\partial x}=0
\end{aligned}
$$

where, $W(x)$ is the flexural displacement; $\omega$ is the angular frequency; $N_{k}, N_{K}, N_{m}, N_{c}$ are the total numbers of the linear springs, rotational springs, masses and spring-mass systems attached to the beam respectively; $k_{b, i}$ and $K_{b, i}$ are the stiffness of the linear and rotational springs, respectively, distributing with function $U k_{i}(x)$ and $U K_{i}(x) ; m_{b, i}$ is the distributed mass located at the beam with function $U m_{i}(x)$; the spring-mass system including mass $m_{z, i}$ and stiffness $k_{z, i}$, which is fixed at rang of function $U c_{i}(x)$, are equal to a stiffness $k_{e, i}$ [8]. The $k_{e, i}$ could be written as

$$
k_{e, i}=k_{z, i} \frac{\omega^{2}}{\omega^{2}-k_{z, i} / m_{z, i}},
$$

where $i$ presents the $i$ th attachment in order.

For a set of general boundary conditions, the Rayleigh-Ritz method or other methods have been usually used to find an approximate solution. While an exact solution for beams under such a set of general boundary conditions is rarely reviewed to the knowledge of the author. To overcome this barrier, the displacement function will be sought in the form of series expansions as

$$
W(x)=\sum_{n=0}^{\infty} A_{n} \cos \lambda_{n} x+\sum_{l=1}^{4} c^{l} \xi^{l}(x),\left(\lambda_{n}=n \pi / L \quad 0 \leqslant x \leqslant L\right),
$$

where, $A_{n}, c^{l}$ are coefficients of displacement function and $\xi^{l}(x)$ represent a set of closed-form sufficiently smooth functions defined over $[0, \mathrm{~L}]$, which are described in Appendix A.

Since the limited expression is used to describe the unlimited expressions in Eq. (4), the ability of convergence of series expansion in Eq. (4) was proved mathematically in reference [21]. For the series expansion is directly used in this paper, our attention will be focused on solving the unknown expansion coefficients by setting the assumed solution satisfy the governing equation and boundary conditions.

By substituting the displacement expression of Eq. (4) into the boundary condition of Eq. (1), results of the derivation process are shown in Appendix A and it could be written in matrix form as

$$
H c=Q a,
$$

in which $\boldsymbol{c}=\left[c_{1}, c_{2}, c_{3}, c_{4}\right], \boldsymbol{a}=\left[A_{0}, A_{1} \ldots A_{n}\right], \boldsymbol{H}=\left[\boldsymbol{e}^{1}, \boldsymbol{e}^{2}, \boldsymbol{e}^{3}, \boldsymbol{e}^{4}\right]^{T}, \boldsymbol{Q}=\left[\boldsymbol{f}^{1}, \boldsymbol{f}^{2}, \boldsymbol{f}^{3}, \boldsymbol{f}^{4}\right] . \boldsymbol{e}^{l}$ and $\boldsymbol{f}^{l}$ are $1 \times 4$ matrix, whose elements could be found in Appendix A, respectively.

Substituting Eq. (4) into the governing differential equation, one is able to obtain

$$
\begin{aligned}
\frac{d^{2}}{d x^{2}} & \left(B(x)\left(\sum_{n=0}^{\infty} \lambda_{n}^{2} A_{n} \cos \lambda_{n} x+\sum_{l=1}^{4} c^{l} \frac{d^{2} \xi^{l}(x)}{d x^{2}}\right)\right) \\
& +\left(\sum_{i=1}^{N k} U k_{i}(x) k_{b, i}+\sum_{i=1}^{N c} U c_{i}(x) k_{e, i}-\omega^{2} \sum_{i=1}^{N m} U m_{i}(x) m_{b, i}-M(x) \omega^{2}\right) \\
& \left(\sum_{n=0}^{\infty} A_{n} \cos \lambda_{n} x+\sum_{l=1}^{4} c^{l} \xi^{l}(x)\right)-\sum_{i=1}^{N K} K_{i} \frac{\partial U K_{i}(x)}{\partial x}\left(-\sum_{n=0}^{\infty} A_{n} \lambda_{n} \sin \lambda_{n} x+\sum_{l=1}^{4} c^{l} \frac{d \xi^{l}(x)}{d x}\right)=0
\end{aligned}
$$


$\sum_{l=1}^{4} \frac{d^{4} \xi^{l}(x)}{d x^{4}}, \sum_{l=1}^{4} \frac{d \xi^{l}(x)}{d x}$ and $\sum_{l=1}^{4} \xi^{l}(x)$ in above equation are expanded into Fourier cosine series, Eq. (6) can be transformed to

$$
\begin{aligned}
& \frac{d^{2}}{d x^{2}}\left(B(x) \sum_{n=0}^{\infty}\left(-\lambda_{n}^{2} A_{n}+\sum_{l=1}^{4} c^{l} \overline{\bar{\alpha}}_{n}^{l}\right) \cos \lambda_{n} x\right) \\
& \quad+\left(\sum_{i=1}^{N k} U k_{i}(x) k_{b, i}+\sum_{i=1}^{N c} U c_{i}(x) k_{e, i}-\omega^{2} \sum_{i=1}^{N m} U m_{i}(x) m_{b, i}-M(x) \omega^{2}\right) \\
& \quad \sum_{n=0}^{\infty}\left(A_{n}+\sum_{l=1}^{4} c^{l} \alpha_{n}^{l}\right) \cos \lambda_{n} x-\sum_{i=1}^{N K} K_{i} \frac{d U K_{i}(x)}{d x}\left(-\sum_{n=0}^{\infty} A_{n} \lambda_{n} \sin \lambda_{n} x+\sum_{l=1}^{4} c^{l} \bar{\alpha}_{n}^{l} \cos \lambda_{n} x\right)=0
\end{aligned}
$$

The coefficients of Fourier expansion $\alpha_{n}^{l}, \bar{\alpha}_{n}^{l}, \overline{\bar{\alpha}}_{n}^{l}$ are demonstrated in Appendix A.

Both sides of Eq. (7) multiple $\cos \lambda_{n} x(n=0, \ldots, N-1)$ respectively and integrate from 0 to $L, N$ equations are derived and provided to solve coefficients $A_{n}$ and natural frequencies of the beam. All equations mentioned above can be written in matrix form and demonstrated as

$$
\left[\boldsymbol{K}_{\boldsymbol{z}}-\omega^{2} \boldsymbol{M}_{\boldsymbol{z}}+\left(\sum_{i=1}^{N k} \boldsymbol{k} \boldsymbol{b}_{i} k_{b, i}+\sum_{i=1}^{N c} \boldsymbol{k e}_{i} k_{e, i}-\omega^{2} \sum_{i=1}^{N m} \boldsymbol{m} \boldsymbol{b}_{i} m_{b, i}-\sum_{i=1}^{N K} \boldsymbol{K} \boldsymbol{b}_{i} K_{i}\right)\right] \boldsymbol{a}=0 .
$$

The coefficient matrices in the equation above are defined in Appendix B.

For the nonlinear characteristic of Eq. (8), the matrix must be transformed polynomial matrix eigenvalue to linear form through method of linearization [22]. The eigenvector $\boldsymbol{a}$ are easy to obtain in the solution of linear eigen matrix and the corresponding vector $c$ can be calculated directly by using Eq. (5). Subsequently, the mode shapes can be constructed by substituting $\boldsymbol{a}$ and $\boldsymbol{c}$ in Eq. (4).

\section{Beam carrying spring-mass systems}

The intersection between human and bridge was simulated by the model of beam or plate with spring-mass systems [23]. In the model, the spring-mass system presents human and the beam describes the bridge. To avoid the resonance of the bridge, the natural frequencies of the beam carrying sprung mass are studied in this Section.

In the following discussions, researches on free vibration of beams with spring-mass systems are divided into two parts. The first part is the natural frequencies of beam with lumped and uniform spring-mass systems. The other part is the natural frequencies of beam with non-uniform spring-mass systems. The first part is proposed to verify the accuracy of the model, and the second part achieves to study the effect of spring-mass systems with different distributions on the natural frequencies of the beams.

\subsection{Beam with lumped and uniform spring-mass systems}

To validate the accuracy of the proposed method, the lumped and uniform spring-mass systems are attached to a uniform beam to form the analysis model. a cantilever uniform $1 \mathrm{~m}$ long beam was considered and made of steel with the modulus of elasticity $E=2.1 \times 10^{11} \mathrm{~Pa}$ and density $\rho=7.8 \times 10^{3} \mathrm{~kg} / \mathrm{m}^{3}$ (value was taken from the references). Then, the Eq. (6) is simplified as

$$
\begin{aligned}
E I & \left(\sum_{n=0}^{\infty} \lambda_{n}^{4} A_{n} \cos \lambda_{n} x+\sum_{l=1}^{4} c^{l} \frac{d^{4} \xi^{l}(x)}{d x^{4}}\right) \\
& +\left(\sum_{i=1}^{N k} U k_{i}(x) k_{b, i}+\sum_{i=1}^{N c} U c_{i}(x) k_{e, i}-\omega^{2} \sum_{i=1}^{N m} U m_{i}(x) m_{b, i}-\rho s \omega^{2}\right) \\
& \left(\sum_{n=0}^{\infty} A_{n} \cos \lambda_{n} x+\sum_{l=1}^{4} c^{l} \xi^{l}(x)\right)-\sum_{i=1}^{N K} K_{i} \frac{\partial U K_{i}(x)}{\partial x}\left(-\sum_{n=0}^{\infty} A_{n} \lambda_{n} \sin \lambda_{n} x+\sum_{l=1}^{4} c^{l} \frac{d \xi^{l}(x)}{d x}\right)=0
\end{aligned}
$$


Table 1

The natural frequency coefficients $\beta_{i}$ for a cantilever beam carrying a spring-mass system

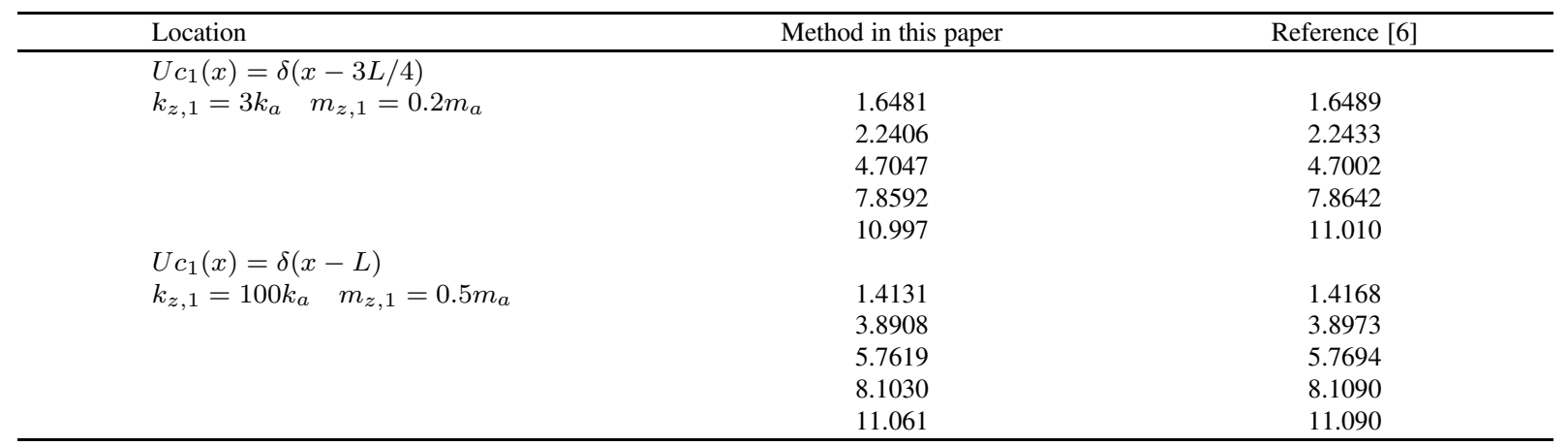

Table 2

The natural frequency coefficients $\beta_{i}$ for a cantilever beam carrying three spring-mass systems

\begin{tabular}{|c|c|c|}
\hline Location & Method in this paper & Reference [6] \\
\hline \multicolumn{3}{|l|}{$U c_{1}(x)=\delta(x-0.1 L) \quad U c_{2}(x)=\delta(x-0.4 L) \quad U c_{2}(x)=\delta(x-0.8 L)$} \\
\hline$k_{z, 1}=3 k_{a} \quad m_{z, 1}=0.2 m_{a}$ & 1.2651 & 1.2651 \\
\hline$k_{z, 2}=4.5 k_{b} \quad m_{z, 2}=0.5 m_{a}$ & 1.7139 & 1.7142 \\
\hline$k_{z, 3}=6 k_{a} \quad m_{z, 3}=m_{a}$ & 1.9678 & 1.9676 \\
\hline & 2.3317 & 2.3314 \\
\hline & 4.7152 & 4.7153 \\
\hline
\end{tabular}

It is easier to obtain the natural frequencies of the beam by analyzing the process in Section 2 .

In the first case, there is only one spring-mass system fitting to the beam at three quarters and the end of the beam. The dimensional and physical properties for the cantilever beam with spring-mass systems are the same as the configuration previously studied in ref. [15]. Then, the distribution functions $U_{c}(x)$ of the spring-mass system are set as $\delta(x-3 L / 4)$ and $\delta(x-L)$ respectively, and the according parameters of the spring-mass systems are $k_{z, 1}=3 k_{a}, m_{z, 1}=0.2 m_{a}$ and $k_{z, 1}=100 k_{a}, m_{z, 1}=0.5 m_{a}$. Where, $m_{a}=\rho s L=15.3875 \mathrm{~kg}, k_{a}=E I / L^{3}=$ $6.34761 \times 10^{4} \mathrm{~N} / \mathrm{m}$. Since $m_{a}$ and $k_{b}$ are the important mass parameter and spring parameter of the cantilever beam respectively, they are used as the bases of dimensionless parameters $m_{z, i}$ and $k_{z, i}$ in the following discussions.

The lowest five natural frequency coefficients $\beta_{i}=L \sqrt{\omega_{i} \sqrt{\rho s / E I}}$ of the cantilever beam obtained from the method in this paper are very close to those from the reference [6], as shown in Table 1.

In the second case, the three sprung masses are located at $x_{1}=0.1 L, x_{2}=0.4 L$ and $x_{3}=0.8 L$ of the cantilever beam. The lowest five natural frequency coefficients of the constrained cantilever beam are shown in Table 2 , from which one sees that values of natural frequency coefficients can be obtained by this method in this paper is almost the same as those in reference [6].

Although the beam carrying lumped attachments is a special situation of the beam carrying distributed attachments when the Dirac delta function $\delta(x)$ is considered as a distribution function, it is not fully enough to prove that the current method could be used to solve the problem of a beam fixed with distributed attachments. So, a simply-simply supported uniform beam with up to three segments of uniformly distributed spring-mass is investigated in detail. The mass and stiffness of the spring-mass are constants in each segment, but vary from one segment to another. Hence, the three distribution functions of spring-mass system can be written commonly as

$$
H_{j}\left(r_{j}^{2}-\left(x-c_{j}\right)^{2}\right)=\left\{\begin{array}{ll}
1 & x_{j}^{1} \leqslant x \leqslant x_{j}^{2} \\
0 & x<x_{j}^{1} \quad x>x_{j}^{2}
\end{array},\right.
$$

where, $r_{j}=\left(x_{j}^{2}-x_{j}^{1}\right) / 2, c_{j}=\left(x_{j}^{2}+x_{j}^{1}\right) / 2, x_{j}^{1}, x_{j}^{2}$ are lower and higher limits of the jth segment respectively.

The structural parameters of simply-simply supported beam are set as below: $s=2 \times 10^{-4} \mathrm{~m}^{2}, I=b h^{3} / 12=$ $1.6667 \times 10^{-9} \mathrm{~m}^{4}, m_{d}=\rho s=1.56 \mathrm{~kg} / \mathrm{m}, k_{d}=E I / L^{4}=3.5 \times 10^{2} \mathrm{~N} / \mathrm{m}^{2}, k_{0}=k_{L}=1 \times 10^{11}, K_{0}=K_{L}=0$. $m_{d}$ and $k_{d}$ are dimensionless parameters used as basement of $m_{z, i}$ and $k_{z, i}$ in the following discussions. In the 
Table 3

The groups of dimensionless natural frequencies coefficients $\beta_{i}$ for a simply-simply supported beam with three segments of uniformly distributed stiffness-masses system

\begin{tabular}{ccccr}
\hline Order of group & First (reference [8]) & Second (reference [8]) & Third (reference [8]) & Fourth (reference [8]) \\
\hline 1 & $1.8857(1.8858)$ & $2.7174(2.717)$ & $3.5321(3.5318)$ & $5.1333(5.1333)$ \\
2 & $2.6391(2.6391)$ & $3.1106(3.111)$ & $3.7465(3.7465)$ & $6.7617(6.7612)$ \\
3 & $2.6575(2.6575)$ & $3.1575(3.1575)$ & $3.7587(3.7587)$ & $9.5759(9.5727)$ \\
4 & $2.6589(2.6588)$ & $3.1613(3.1613)$ & $3.7603(3.7601)$ & $12.643(12.629)$ \\
5 & $2.659(2.6591)$ & $3.162(3.162)$ & $3.7605(3.7604)$ & $15.763(15.74)$ \\
6 & $2.6591(2.6591)$ & $3.1622(3.1623)$ & $3.7606(3.7605)$ & $18.844(18.868)$ \\
\hline
\end{tabular}

simulation, the sprung mass occupies the full length of the beam and the lengths of the three segments are the same. Accordingly, the distribution function are $H_{1}\left(1 / 36-(x-1 / 6)^{2}\right), H_{2}\left(1 / 36-(x-1 / 2)^{2}\right), H_{2}(1 / 36-(x-$ $\left.5 / 6)^{2}\right)$ separately. The spring-masses have the parameters $k_{z, 1}=500 k_{d}$ and $m_{z, 1}=2.5 m_{d}$ on the first segment, $k_{z, 2}=500 k_{d}$ and $m_{z, 2}=5 m_{d}$; on the second one, $k_{z, 3}=500 k_{d}$ and $m_{z, 3}=10 m_{d}$ on the third one. As the dimensionless natural frequencies in ref. [8] are the square of natural frequency coefficients $\beta_{i}$ in this paper, results are extracted from the reference and filled in Table 3. In Table 3, the excellent agreement is achieved by comparing outcomes from the current method and reference.

From the comparisons between results from the method in this paper and reference in Tables 1-3, it is obviously demonstrated that natural frequencies of the beam carrying lumped and uniform distribution sprung mass are very close to those in references. While, there are several different methods used in the references to obtain the natural frequencies and only one general method is introduced with the same results in this paper. Hence, the proposed method is useful and accurate approach for analyzing the free vibration of the beam carrying spring-mass systems.

\subsection{Beam carrying non-uniform spring-mass systems}

In the above analysis, the attachment distribution function is uniform along the length of beam at every segment, which simulates the humans staying at bridge with unchanged distribution. While human stay at bridge with arbitrary distribution in fact, it is important to analyze free vibration of beam carrying non-uniform spring-mass system theoretically.

In this part, five different distributions of spring-mass systems, which are rectangular, triangle, sine, linear and exponential style respectively, are introduced to study their effects on changing the natural frequencies of the uniform beam. The sketch map of these five distributions is plotted in Fig. 2.

To keep spring-mass system on the beam as the same number, the function of these different distributions are accordingly listed as

$$
\begin{aligned}
& D(x)=\left\{\begin{array}{ll}
\frac{1}{0.5 L} & 0.25 L<x<0.75 L \\
0 & 0<x<0.25 L, 0.75 L<x<L
\end{array},\right. \\
& D(x)=\left\{\begin{array}{ll}
\frac{4}{L} x & 0<x<0.5 L \\
-\frac{4}{L}\left(x-\frac{L}{2}\right)+2 & 0.5 L<x<L
\end{array},\right. \\
& D(x)=\frac{\pi}{2 L} \sin \frac{\pi}{L} x \quad 0<x<L, \\
& D(x)=-\frac{2}{L} x+2 \quad 0<x<L,
\end{aligned}
$$

and

$$
D(x)=\frac{e^{-x}}{1-e^{-1}} \quad 0<x<L
$$

The stiffness $k_{z}$ and weight $m_{z}$ of spring and mass are set as $500 k_{a}, 1600 k_{a}$ and $5 m_{a}, m_{a}$ respectively. The dimensionless natural frequencies coefficients $\beta_{i}$ of the beam carrying these distributions' spring-mass systems 
Table 4

Dimensionless modal frequencies of simply-simply supported beams carrying different distributed spring-mass systems

\begin{tabular}{|c|c|c|c|c|c|c|c|c|c|c|}
\hline \multirow{2}{*}{$\begin{array}{l}\text { Mode } \\
\text { Order }\end{array}$} & \multicolumn{2}{|c|}{ Rectangular } & \multicolumn{2}{|c|}{ Linear } & \multicolumn{2}{|c|}{ Exponential } & \multicolumn{2}{|c|}{ Triangle } & \multicolumn{2}{|c|}{ Sine } \\
\hline & $\begin{array}{l}\text { First } \\
\text { group }\end{array}$ & $\begin{array}{c}\text { Second } \\
\text { group }\end{array}$ & $\begin{array}{l}\text { First } \\
\text { group }\end{array}$ & $\begin{array}{l}\text { Second } \\
\text { group }\end{array}$ & $\begin{array}{l}\text { First } \\
\text { group }\end{array}$ & $\begin{array}{c}\text { Second } \\
\text { group }\end{array}$ & $\begin{array}{l}\text { First } \\
\text { group }\end{array}$ & $\begin{array}{l}\text { Second } \\
\text { group }\end{array}$ & $\begin{array}{l}\text { First } \\
\text { group }\end{array}$ & $\begin{array}{l}\text { Second } \\
\text { group }\end{array}$ \\
\hline \multicolumn{11}{|c|}{$k_{z}=500 k_{a}, m_{z}=5 m_{a}$} \\
\hline 1 & 1.7634 & 5.6092 & 1.9405 & 5.0625 & 2.2651 & 4.3733 & 1.8188 & 5.4514 & 1.8383 & 5.3995 \\
\hline 2 & 2.9331 & 6.7488 & 2.9579 & 6.7710 & 3.1467 & 6.2871 & 2.9392 & 6.7563 & 2.9277 & 6.7836 \\
\hline 3 & 3.1292 & 9.5463 & 3.1194 & 9.5767 & 3.1598 & 9.4348 & 3.1157 & 9.5837 & 3.1139 & 9.5784 \\
\hline 4 & 3.1546 & 12.632 & 3.1488 & 12.642 & 3.1614 & 12.571 & 3.1480 & 12.643 & 3.1473 & 12.633 \\
\hline \multicolumn{11}{|c|}{$k_{z}=1600 k_{a}, m_{z}=m_{a}$} \\
\hline 1 & 2.4560 & 7.9414 & 2.6302 & 7.3245 & 2.8810 & 6.4000 & 2.5131 & 7.8518 & 2.5323 & 7.8220 \\
\hline 2 & 4.9317 & 7.9536 & 4.9833 & 8.1371 & 6.0902 & 6.9028 & 4.9454 & 8.0177 & 4.9102 & 8.0809 \\
\hline 3 & 6.0893 & 9.9023 & 6.0204 & 9.9636 & 6.3104 & 9.4589 & 6.0015 & 9.890 & 5.9920 & 9.9721 \\
\hline 4 & 6.2730 & 12.876 & 6.2340 & 12.788 & 6.3239 & 12.571 & 6.2300 & 12.788 & 6.2253 & 12.781 \\
\hline
\end{tabular}

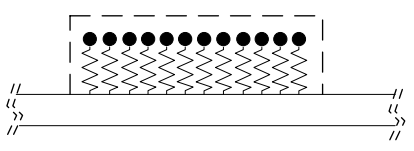

(a) Rectangular style

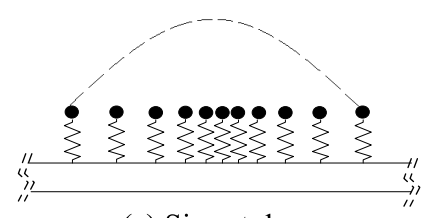

(c) Sine style

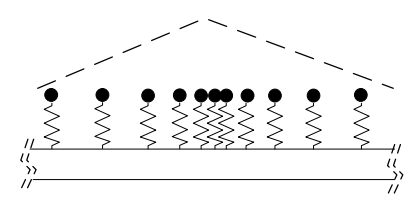

(b) Triangle style

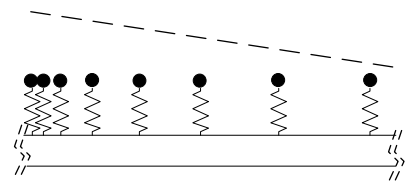

(d) Sine style

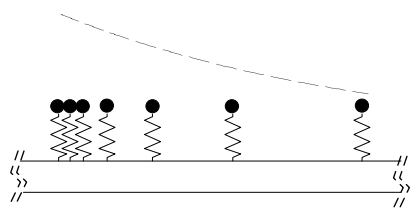

(e) Exponential style

Fig. 2. Sketch of five distributions.

with simply-simply supported boundary conditions are calculated and listed in Table 4. It is easy to reveal that the spring-mass system with rectangular and exponential distribution makes greatest and fewest changes on the natural frequency of the first mode respectively. When the order of mode turns to the second, distribution of spring-mass system which makes greatest variations on the natural frequency is changed to linear style. For other modes, the effects of spring-mass system with different distributions on the change of beam's natural frequencies are almost same.

To expend range of discussion, the case of stiffness and weight set as $500 k_{a}$ and $5 m_{a}$ is selected and analyzed with free-clamped boundary condition. The first four modal frequencies are obtained and listed in Table 5. From the results, it is shown that the distribution of the most effective spring-mass system for changing first natural frequency of the beam is linear style, the next one is exponential style and the least effective case is rectangular style. For the second modal frequencies, the distribution of the most effective spring-mass system becomes exponential style and the least one changes to sine style. In other modes, the effects of spring-mass system with different distributions on their natural frequencies' change are close to each other.

In above analysis, it seems that the most effective distribution of spring-mass system varies with the order of modes and boundary conditions and there is no principle about this variation. When the mode shapes of the beam 
Table 5

Dimensionless modal frequencies of free-clamped beams carrying different distributed spring-mass systems

\begin{tabular}{|c|c|c|c|c|c|c|c|c|c|c|}
\hline $\begin{array}{l}\text { Mode } \\
\text { Order }\end{array}$ & \multicolumn{2}{|c|}{ Rectangular } & \multicolumn{2}{|c|}{ Linear } & \multicolumn{2}{|c|}{ Exponential } & \multicolumn{2}{|c|}{ Triangle } & \multicolumn{2}{|c|}{ Sine } \\
\hline 2 & 2.7572 & 6.0741 & 2.5685 & 5.8940 & 2.7281 & 6.4062 & 2.6448 & 5.9057 & 2.6449 & 5.8382 \\
\hline 3 & 3.1170 & 8.153 & 3.0663 & 8.1276 & 3.1552 & 8.4357 & 3.0789 & 8.1119 & 3.0755 & 8.1192 \\
\hline 4 & 3.1532 & 11.079 & 3.1377 & 11.099 & 3.1609 & 11.163 & 3.1400 & 11.100 & 3.1391 & 11.093 \\
\hline
\end{tabular}

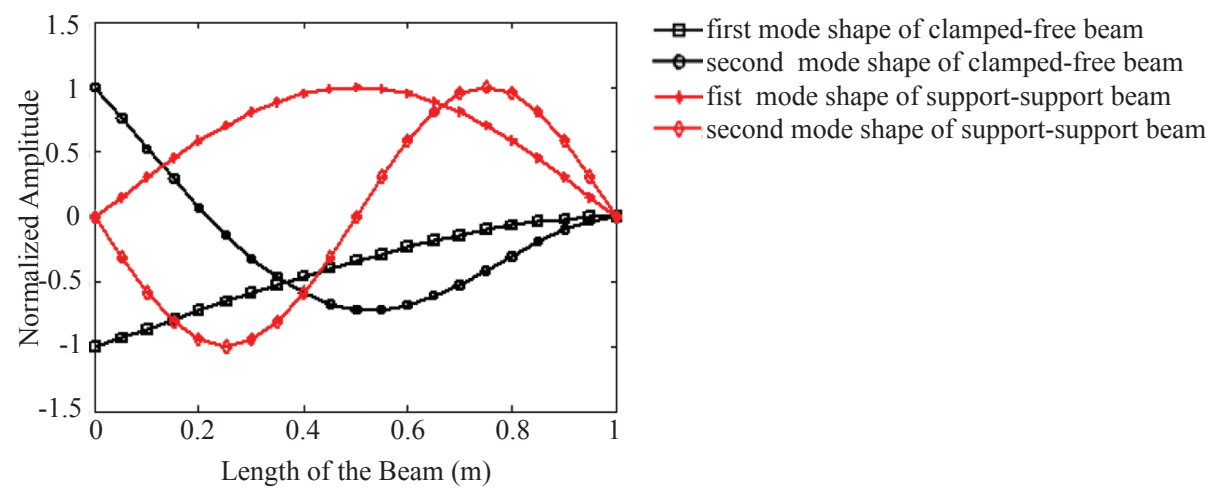

Fig. 3. Mode shapes of simply-simply supported and clamped-free beam without spring-mass system.

with clamped-free and simply-simply supported boundary conditions are drawn in Fig. 3 and compared with the sketch of five different distributions, a general rule is observed and shown that the distribution density of springmass system at the peaks of the mode shapes is a key factor for its effect on the change of beam's natural frequencies.

For the simply-simply supported beam, it is clearly seen that the distribution density of rectangular and linear style is the most concentrated at the peaks of the first and second mode shape respectively. In the case of free-clamped beam, the distribution density of linear and exponential style at the peaks of the first and second mode shape is higher than those of other styles. In these situations, the effects of spring-mass systems on the change of beam's natural frequencies reach the highest level. So that, the more concentrated the distribution density locates at the peaks of the mode shape of the beam, the greater effects is taken on its natural frequencies.

While the peaks of the mode shape grow with the increase of the order of the mode, the average value of distribution density of different styles at these positions gets close to each other. Therefore, the effects of spring-mass system with different distributions on the natural frequencies' change are near to each other.

\section{Beams resting on Pasternak soil}

The model of the beam resting on Pasternak soil is applied to simulate the situation of pipe seating on the elastic foundation. In the author's view, the influences of soil on the beam could be considered as a type of attachment. So, the proposed method can also be used to analyze the problem of free vibration of Euler beam resting on Pasternak soil. According to a beam with flexible ends resting on Pasternak soil in reference [13], the influences of two parameters elastic soil are equal to shear and linear elastic springs, which connect to beam from one end to the other end. The equation of beam can be easily deduced by means of Hamilton's principle as

$$
\frac{\partial^{2}}{\partial x^{2}}\left(B(x) \frac{\partial^{2} W}{\partial x^{2}}\right)-\bar{k}_{1} \frac{\partial^{2} W(x)}{\partial x^{2}}+\left(\bar{k}_{0}-M(x) \omega^{2}\right) W=0
$$


Table 6

Numerical comparisons with reference [14] for a clamped-clamped beam on Pasternak soil

\begin{tabular}{|c|c|c|c|c|c|c|}
\hline$\Omega_{\Omega_{2}} \Omega_{1}$ & $\begin{array}{c}0 \\
\text { This paper }\end{array}$ & $\begin{array}{c}0 \\
\text { Reference [14] }\end{array}$ & $\begin{array}{c}1 \\
\text { This paper }\end{array}$ & $\begin{array}{c}1 \\
\text { Reference [14] }\end{array}$ & $\begin{array}{c}2.5 \\
\text { This paper }\end{array}$ & $\begin{array}{c}2.5 \\
\text { Reference [14] }\end{array}$ \\
\hline \multirow[t]{3}{*}{0} & 4.73 & 4.73 & 4.9925 & 4.994 & 5.3183 & 5.32 \\
\hline & 7.8531 & 7.854 & 8.0774 & 8.078 & 8.3808 & 8.381 \\
\hline & 10.995 & 10.996 & 11.174 & 11.174 & 11.427 & 11.43 \\
\hline \multirow[t]{3}{*}{100} & 4.9504 & 4.95 & 5.1823 & 5.182 & 5.4772 & 5.477 \\
\hline & 7.9042 & 7.904 & 8.1244 & 8.124 & 8.423 & 8.423 \\
\hline & 11.014 & 11.014 & 11.192 & 11.192 & 11.444 & 11.444 \\
\hline \multirow[t]{3}{*}{100000} & 10.123 & 10.123 & 10.152 & 10.152 & 10.194 & 10.194 \\
\hline & 10.839 & 10.839 & 10.927 & 10.927 & 11.055 & 11.055 \\
\hline & 12.526 & 12.526 & 12.648 & 12.648 & 12.825 & 12.825 \\
\hline
\end{tabular}

where, $\bar{k}_{0}$ the Winkler modulus of the subgrade reaction, $\bar{k}_{1}$ is the second foundation parameter. Substituting Eq. (4) into Eq. (16), the governing differential function can be transformed to

$$
\begin{aligned}
& \frac{d^{2}}{d x^{2}}\left(B(x)\left(\sum_{n=0}^{\infty}-\lambda_{n}^{2} A_{n} \cos \lambda_{n} x+\sum_{l=1}^{4} c^{l} \frac{d^{2} \xi^{l}(x)}{d x^{2}}\right)\right)-\bar{k}_{1}\left(-\sum_{n=0}^{\infty} A_{n} \lambda_{n}^{2} \cos \lambda_{n} x+\sum_{l=1}^{4} c^{l} \frac{d^{2} \xi^{l}(x)}{d x^{2}}\right), \\
& \quad+\left(\bar{k}_{0}-M(x) \omega^{2}\right)\left(\sum_{n=0}^{\infty} A_{n} \cos \lambda_{n} x+\sum_{l=1}^{4} c^{l} \xi^{l}(x)\right)=0
\end{aligned}
$$

which could be written in matrix form

$$
\left(K_{z}+\bar{k}_{0} M_{z}-\bar{k}_{1} C_{z}-\omega^{2} M_{z}\right) a=0 .
$$

The matrix $C_{z}$ is defined in Appendix C.

The natural frequencies are calculated by solving the eigenvalue problem of governing equation, while the mode shapes are derived from eigenvector combining with relationship between $\boldsymbol{a}$ and $\boldsymbol{c}$ in Eq. (5). After changing right side of Eq. (2) to force vector, forced vibration problem of beam resting on Pasternak soil can also be solved.

\section{Uniform beam on Pasternak soil}

For validating the accuracy of the model established by using the proposed method in this paper, the case of a uniform beam resting on Pasternak soil is selected and analyzed. The parameters of material and geometry of the uniform beam is in step with those in reference [13]. Before the calculation, non-dimensional soil parameter coefficients are set as

$$
\Omega_{0}=k_{0} L^{4} / E I, \Omega_{1}=k_{1} L^{2} / E I \pi^{2} .
$$

The dimensionless natural frequencies $\beta_{i}$ obtained by the current method in this paper are listed with those in reference [14] in Table 6, which show that the results of two approaches have excellent agreement.

The examples of uniform beams above in Tables 1-6 are presented as special cases of beam carrying any type of attachments with elastically restrained boundary. It is shown that the frequency parameters for different type of attachments, distributions and boundary conditions can be accurately determined by modifying the parameters of attachments, the distribution functions and the stiffness of the restraining springs accordingly. It should be emphasized that unlike most existing approaches, the current method provides a unified solution for a variety of attachments with different distributions and boundary conditions. The change from one case to another is as simple as modifying the material properties or structural dimension. 


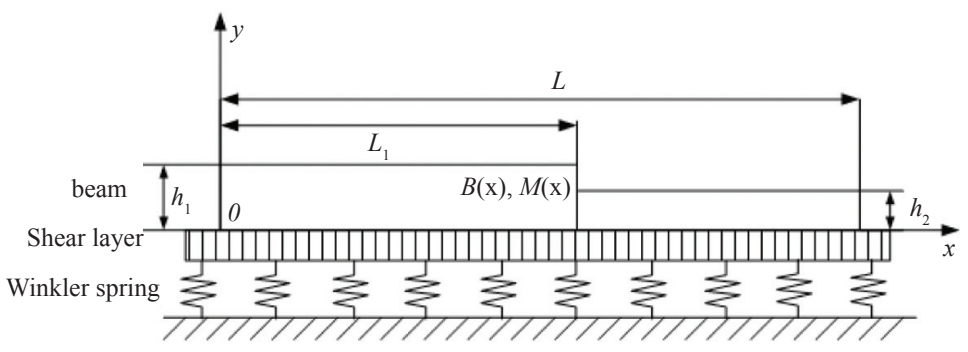

Fig. 4. The sketch of one stepped beam on Pasternak soil.

\section{Non-uniform beam on Pasternak soil}

In the real pipe-like structures staying on Pasternak soil, the shape of the cross section is not limited to uniform style and the non-uniform shapes of the cross section often occur. So, the researches on free vibration of beams resting on Pasternak soil should not be constrained in the case of uniform cross section and studies about free vibration of non-uniform beam resting on Pasternak soil need be taken in consideration. In this Section, one stepped beam is selected as an example of non-uniform beam to study its free vibration by using the proposed method in this paper, and the sketch of the whole model is draw in Fig. 4.

In the Fig. 4, the bending stiffness and mass function of one stepped beam are described as

$$
B(x)= \begin{cases}E I_{1} & 0<x<L_{1} \\ E I_{2} & L_{1}<x<L\end{cases}
$$

and

$$
M(x)= \begin{cases}\rho s_{1} & 0<x<L_{1} \\ \rho s_{2} & L_{1}<x<L\end{cases}
$$

where, $I_{1}, I_{2}$ are the moment of inertia of cross sections at two parts of one stepped beam respectively, $s_{1}, s_{2}$ present the area of cross sections at two different steps of the beam, $L_{1}, L$ describe the length of the first step and the whole length of the beam. The step ratio $\lambda$ and location $R_{1}$ of the beam [23] are defined as

$$
\left\{\begin{array}{l}
\lambda=\frac{L_{1}}{L} \\
R_{1}=\frac{h_{2}}{h_{1}}
\end{array}\right.
$$

where, $h_{1}$ and $h_{2}$ present the thickness of the first and second part of one stepped beam.

In the analysis, the boundary condition and material parameters are selected the same as those of the uniform beam. The value of the step ratio $\lambda$ and location $R_{1}$ are set as 0.5 and 0.25 . Thought the solution of the Eqs (12)(14) above, the dimensionless natural frequencies of the stepped beam resting on Pasternak soil with different soil parameters are calculated and listed in Table 7.

When the non-dimensional soil parameter coefficients are considered as 0 , the model of one stepped beam testing on Pasternak soil is simplified to the model of the one stepped beam without attachments. Thus, the accuracy of the model of the non-uniform beam is proved by little difference between the results derived from the proposed method in this paper and those in reference [24].

Comparing with the results of natural frequencies in Table 6, the natural frequencies of the stepped beam more easily increase with the growth of non-dimensional soil parameter coefficients. In the view of the geometry of the beam, the one stepped beam lost a part of structure comparing with the uniform beam and the effect of missing part on stiffness of the structure is greater than that on the mass. So, the natural frequencies of one stepped beam are lower than those of uniform beam in the 'vacuo' situation. While the stiffness of the whole beam increases with the growth of non-dimensional soil parameter coefficients, the effect of the loss stiffness become limited and the miss 
Table 7

Dimensionless natural frequencies of one stepped beam on Pasternak soil with clamped-clamped boundary condition

\begin{tabular}{cccccc}
\hline$\Omega_{2}$ & $\Omega_{1}$ & & 0 & 1 & 2.5 \\
\cline { 3 - 5 } & & This paper & Reference [14] & & 6.1654 \\
& 3.9912 & 3.9906 & 6.1618 & 7.2611 & 8.6724 \\
& 6.1624 & 8.3054 & - & 9.6437 & 10.196 \\
\multirow{2}{*}{100} & 8.3091 & - & 5.6509 & 6.4126 \\
& 4.7797 & - & 7.7229 & 8.8052 \\
& 6.5226 & - & 9.7414 & 10.982 \\
\multirow{2}{*}{100000} & 8.4713 & - & 12.33 & 12.351 \\
& 12.148 & - & 14.098 & 14.505 \\
& 13.742 & 14.403 & 15.502 & 15.513 \\
\hline
\end{tabular}

of the mass in the beam takes dominate part in its natural frequencies. The natural frequencies of the stepped beam become higher than those of uniform beam with large non-dimensional soil parameter coefficients.

From above discussions, it is easy to conclude that the natural frequencies of the stepped beam resting on Pasternak soil grow greatly with the increase of the soil parameters. In some situations, the stepped beam is used to approximate the non-uniform beam with various shapes of cross sections. Hence, the principle obtained in stepped beam could be also used to describe those of non-uniform beam.

\section{Conclusion}

In this paper, a new analytic method is proposed and applied to solve free vibration of constrained beams carrying any type of attachments with arbitrary distribution. Through three different simulation examples which involve beams attached by lumped and uniform spring-masses and resting on Pasternak soil separately, the natural frequencies and relevant coefficients are calculated and compared with those in the references. These comparisons show great agreement and give evidences that the current method is accurate for analyzing the free vibration of the constrained beam carrying attachments with arbitrary distributions. Furthermore, the natural frequencies of uniform beam with different distributions are calculated and reveal that the distribution density of spring-mass system at the peaks of the beam's mode shape is the key factor for its effect on the change of their natural frequencies. Then, the natural frequencies of one stepped beam resting on Pasternak soil are analyzed and proved to be more sensitive to the change of non-dimensional soil parameter coefficients.

In brief, the analytical method proposed in this paper is a general and important approach for analyzing the free vibration of constrained beams carrying any type attachments with arbitrary distributions. It can be not only applied to deal with problems in the references, but also be extended to analyze free vibration of the beam fixed with attachments which distributes various with its length and non-uniform beam resting on Pasternak soil.

\section{Acknowledgments}

This research is financially supported by the NPU Foundation for Fundamental Research, Ministry of Education Fund for Doctoral Students Newcomer Awards of china. Author wish to thank these supports.

\section{Appendix}

\section{A. Parameters in section of theory}

The $\zeta^{l}(x)$ is considered as

$$
\xi^{l}(x)=s_{1}^{l} \sin \left(\frac{\pi x}{2 L}\right)+s_{2}^{l} \cos \left(\frac{\pi x}{2 L}\right)+s_{3}^{l} \sin \left(\frac{3 \pi x}{2 L}\right)+s_{4}^{l} \cos \left(\frac{3 \pi x}{2 L}\right), \quad l=1 \ldots 4,
$$


where

$$
\left\{\begin{array}{l}
s_{1}^{1}=\frac{9 L}{4 \pi} \\
s_{2}^{1}=0 \\
s_{3}^{1}=-\frac{L}{12 \pi} \\
s_{4}^{1}=0
\end{array}, \quad\left\{\begin{array}{l}
s_{1}^{2}=0 \\
s_{2}^{2}=-\frac{9 L}{4 \pi} \\
s_{3}^{2}=0 \\
s_{4}^{2}=-\frac{L}{12 \pi}
\end{array}, \quad\left\{\begin{array}{l}
s_{1}^{3}=\frac{L^{3}}{\pi^{3}} \\
s_{2}^{3}=0 \\
s_{3}^{3}=-\frac{L^{3}}{3 \pi^{3}} \\
s_{4}^{3}=0
\end{array}, \quad\left\{\begin{array}{l}
s_{1}^{4}=0 \\
s_{2}^{4}=-\frac{L^{3}}{\pi^{3}} \\
s_{3}^{4}=0 \\
s_{4}^{4}=-\frac{L^{3}}{3 \pi^{3}}
\end{array} .\right.\right.\right.\right.
$$

The relationship between $c_{l}$ and $A_{n}$ is written as

$$
\begin{aligned}
& \sum_{l=1}^{4}\left[c^{l}\left(\overline{k_{0}} \xi^{l}(0)+\xi^{\prime \prime \prime l}(0)\right)\right]=-\overline{k_{0}} \sum_{n=0}^{\infty} A_{n}, \\
& \sum_{l=1}^{4}\left[c^{l}\left(\overline{K_{0}} \xi^{\prime l}(0)-\xi^{\prime \prime l}(0)\right)\right]=-\lambda_{n}^{2} \sum_{n=0}^{\infty} A_{n}, \\
& \sum_{l=1}^{4}\left[c^{l}\left(\overline{k_{L}} \xi^{l}(L)-\xi^{\prime \prime \prime l}(L)\right)\right]=-\overline{k_{L}} \sum_{n=0}^{\infty} A_{n}(-1)^{n}
\end{aligned}
$$

and

$$
\sum_{l=1}^{4}\left[c^{l}\left(\overline{K_{L}} \xi^{\prime l}(L)+\xi^{\prime \prime l}(L)\right)\right]=\lambda_{n}^{2} \sum_{n=0}^{\infty} A_{n}(-1)^{n}
$$

The matrix in Eq. (7) are described as

$$
\left\{\begin{array}{l}
e_{m}^{1}=\overline{k_{0}} \xi^{m}(0)+\xi^{m \prime \prime \prime}(0) \\
e_{m}^{2}=\overline{K_{0}} \xi^{m^{\prime}}(0)-\xi^{m \prime \prime}(0) \\
e_{m}^{3}=\overline{k_{l}} \xi^{m}(l)-\xi^{m \prime \prime \prime}(l) \\
e_{m}^{4}=\overline{K_{l}} \xi^{m^{\prime}}(l)-\xi^{m \prime \prime}(l)
\end{array}, \quad\left\{\begin{array}{l}
f_{d}^{1}=-\overline{k_{0}} \\
f_{d}^{2}=-\lambda_{d}^{2} \\
f_{d}^{3}=-\overline{k_{l}}(-1)^{d} \cdot \quad m=1 \ldots 4, d=1 \ldots N \\
f_{d}^{4}=\lambda_{n}^{2}(-1)^{d}
\end{array}\right.\right.
$$

The detail expression of $\alpha_{n}^{l}, \bar{\alpha}_{n}^{l}, \overline{\bar{\alpha}}_{n}^{l}$ is shown [21] as

$$
\alpha_{n}^{l}=\sum_{p=1}^{4} s_{p}^{l} \tau_{n}^{p}, \quad \bar{\alpha}_{n}^{l}=\sum_{p=1}^{4} s_{p}^{l} \sigma_{n}^{p}\left(\lambda_{p}\right), \quad \overline{\bar{\alpha}}_{n}^{l}=\sum_{p=1}^{4} s_{p}^{l}\left(\lambda_{p}\right)^{4} \tau_{n}^{p},
$$

where, $\tau_{n}^{p}$ and $\sigma_{n}^{p}$ denote the expansion coefficients of the following functions:

$$
\begin{gathered}
\tau_{n}^{1}=\left\{\begin{array}{ll}
n=0 & \frac{2}{\pi} \\
n \neq 0 & \frac{4}{\left(1-4 n^{2}\right) \pi}
\end{array}, \quad \tau_{n}^{2}= \begin{cases}n=0 & \frac{2}{\pi} \\
n \neq 0 & \frac{4(-1)^{n}}{\left(1-4 n^{2}\right) \pi},\end{cases} \right. \\
\tau_{n}^{3}=\left\{\begin{array}{lll}
n=0 & \frac{2}{3 \pi} \\
n \neq 0 & \frac{12}{\left(9-4 n^{2}\right) \pi}
\end{array}, \quad \tau_{n}^{4}= \begin{cases}n=0 & -\frac{2}{3 \pi} \\
n \neq 0 & \frac{12(-1)^{n+1}}{\left(9-4 n^{2}\right) \pi}\end{cases} \right. \\
\sigma_{n}^{1}=\left\{\begin{array}{lll}
n=0 & \frac{2}{\pi} \\
n \neq 0 & \frac{4(-1)^{n}}{\left(1-4 n^{2}\right) \pi}
\end{array}, \quad \sigma_{n}^{2}=\left\{\begin{array}{ll}
n=0 & -\frac{2}{\pi} \\
n \neq 0 & -\frac{4}{\left(1-4 n^{2}\right) \pi}
\end{array},\right.\right. \\
\sigma_{n}^{3}=\left\{\begin{array}{lll}
n=0 & -\frac{2}{3 \pi} \\
n \neq 0 & \frac{12(-1)^{n+1}}{\left(9-4 n^{2}\right) \pi}
\end{array},\right.
\end{gathered}
$$


B. Definitions of coefficient matrices in Eq. (13)

The matrix of $\boldsymbol{B}, \boldsymbol{F}$ and $\boldsymbol{D}$ are defined as

$$
\left\{\begin{array} { l } 
{ B _ { 1 } = ( z a ^ { 1 } , z a ^ { 2 } , z a ^ { 3 } , z a ^ { 4 } ) } \\
{ B _ { 2 } = ( z d ^ { 1 } , z d ^ { 2 } , z d ^ { 3 } , z d ^ { 4 } ) }
\end{array} \quad \text { and } \quad \left\{\begin{array}{l}
F=\left(z b^{1}, z b^{2}, z b^{3}, z b^{4}\right) \\
D=\left(z c^{1}, z c^{2}, z c^{3}, z c^{4}\right)
\end{array}\right.\right.
$$

where, $\boldsymbol{z} \boldsymbol{a}^{l}, \boldsymbol{z} \boldsymbol{b}^{l}$ and $\boldsymbol{z} \boldsymbol{c}^{l}$ are $N \times 1$ matrices, they can be written as

$$
\left\{\begin{aligned}
\boldsymbol{z} \boldsymbol{a}_{n}^{l} & =\left.\sum_{m=1}^{M} \frac{d B(x)}{x} \cos \lambda_{m-1} x\right|_{0} ^{L} \bar{\alpha}_{m}^{l} \cos \lambda_{n-1} x+\left.\sum_{m=1}^{M} B(x) \cos \lambda_{m-1} x\right|_{0} ^{L} \overline{\bar{\alpha}}_{m}^{l} \cos \lambda_{n-1} x \\
& +\left.\sum_{m=1}^{M} B(x)\left(-\lambda_{n-1}\right) \cos \lambda_{m-1} x\right|_{0} ^{L} \bar{\alpha}_{m}^{l} \sin \lambda_{n-1} x \\
& +\sum_{m=1}^{M} \int_{0}^{L}\left(B(x) \bar{\alpha}_{m}^{l}\left(-\lambda^{2}{ }_{n-1}\right) \cos \left(\lambda_{m-1} x\right) \cos \left(\lambda_{n-1} x\right)\right) d x \\
\boldsymbol{z} \boldsymbol{d}_{n}^{l} & =\sum_{m=1}^{M} \int_{0}^{L}\left(M(x) \alpha_{m}^{l} \cos \left(\lambda_{m-1} x\right) \cos \left(\lambda_{n-1} x\right)\right) d x
\end{aligned}\right.
$$

and

$$
\left\{\begin{array}{l}
\boldsymbol{z} \boldsymbol{b}_{n}^{l}=\alpha_{n}^{l} \\
\boldsymbol{z} \boldsymbol{c}_{n}^{l}=\bar{\alpha}_{n}^{l}, \quad l=1 \ldots 4 .
\end{array}\right.
$$

To be convenient, setting

$$
\left\{\begin{array} { l } 
{ S R = F H ^ { - 1 } Q } \\
{ S L = D H ^ { - 1 } Q }
\end{array} \quad \text { and } \quad \left\{\begin{array}{l}
K_{z}=K_{t}+B_{1} H^{-1} Q \\
M_{z}=M_{t}+B_{2} H^{-1} Q
\end{array}\right.\right.
$$

where, $\boldsymbol{K}_{\boldsymbol{z}}, \boldsymbol{K} \boldsymbol{z}, \boldsymbol{k} \boldsymbol{b}_{i}, \boldsymbol{k} \boldsymbol{e}_{i}, \boldsymbol{m} \boldsymbol{b}_{i}, \boldsymbol{K} \boldsymbol{b}_{i}$ are $N \times N$ matrices. Their elements are described as

$$
\begin{aligned}
\boldsymbol{K}_{\boldsymbol{t} m, n} & =\left.\frac{d B(x)}{d x} \lambda_{m-1}^{2} \cos \left(\lambda_{m-1} x\right) \cos \left(\lambda_{n-1} x\right)\right|_{0} ^{L}+\left.B(x) \lambda_{m-1}^{3} \cos \left(\lambda_{m-1} x\right) \cos \left(\lambda_{n-1} x\right)\right|_{0} ^{L} \\
& +B(x)-\left.\lambda_{m-1}^{2} \lambda_{n-1} \cos \left(\lambda_{m-1} x\right) \cos \left(\lambda_{n-1} x\right)\right|_{0} ^{L} \\
& +\int_{0}^{L}\left(B(x) \lambda_{m-1}^{2} \lambda^{2}{ }_{n-1} \cos \left(\lambda_{m-1} x\right) \cos \left(\lambda_{n-1} x\right)\right) d x \\
\boldsymbol{M}_{\boldsymbol{z} m, n} & =\int_{0}^{L}\left(M(x) \cos \left(\lambda_{m-1} x\right) \cos \left(\lambda_{n-1} x\right)\right) d x, \\
\boldsymbol{k b}_{i m, n} & =\int_{0}^{L}\left(U k_{i}(x) \cos \left(\lambda_{m-1} x\right) \cos \left(\lambda_{n-1} x\right)\right) d x \\
& +\sum_{s=1}^{N} S R_{s, n}\left[\int_{0}^{L}\left(U k_{i}(x) \cos \left(\lambda_{m-1} x\right) \cos \left(\lambda_{s-1} x\right)\right) d x\right] \\
\boldsymbol{k e}_{i m, n} & =\int_{0}^{L}\left(U c_{i}(x) \cos \left(\lambda_{m-1} x\right) \cos \left(\lambda_{n-1} x\right)\right) d x \\
& +\sum_{s=1}^{N} S R_{s, n}\left[\int_{0}^{L}\left(U c_{i}(x) \cos \left(\lambda_{m-1} x\right) \cos \left(\lambda_{s-1} x\right)\right) d x\right],
\end{aligned}
$$




$$
\begin{aligned}
\boldsymbol{m b}_{i m, n} & =\int_{0}^{L}\left(U m_{i}(x) \cos \left(\lambda_{m-1} x\right) \cos \left(\lambda_{n-1} x\right)\right) d x \\
& +\sum_{s=1}^{N} S R_{s, n}\left[\int_{0}^{L}\left(U m_{i}(x) \cos \left(\lambda_{m-1} x\right) \cos \left(\lambda_{s-1} x\right)\right) d x\right]
\end{aligned}
$$

and

$$
\begin{aligned}
\boldsymbol{K} \boldsymbol{b}_{i m, n} & =\int_{0}^{L}\left(-\lambda_{m-1}\right)\left(U K_{i}(x) \cos \left(\lambda_{m-1} x\right) \sin \left(\lambda_{n-1} x\right)\right) d x \\
& +\sum_{s=1}^{N} S L_{s, n}\left[\int_{0}^{L}\left(U K_{i}(x) \cos \left(\lambda_{m-1} x\right) \cos \left(\lambda_{s} x\right)\right) d x\right]
\end{aligned}
$$

\section{Definitions of matrix $\boldsymbol{C}_{\boldsymbol{z}}$}

The matrix $\boldsymbol{C}_{\boldsymbol{z}}$ is defined as

$$
C_{z}=\bar{M}+Z H^{-1} Q
$$

The elements of $\boldsymbol{Z}$ and $\bar{M}$ are set as

$$
\overline{\boldsymbol{M}}_{n, n}=-\lambda_{n}^{2}, \boldsymbol{D}=\left(\boldsymbol{z} \boldsymbol{z}^{1}, \boldsymbol{z z ^ { 2 }}, \boldsymbol{z \boldsymbol { z } ^ { 3 }}, \boldsymbol{z \boldsymbol { z } ^ { 4 }}\right) .
$$

$\boldsymbol{z} \boldsymbol{z}^{l}$ is $N \times 1$ matrix, it could be written as

$$
\boldsymbol{z} \boldsymbol{z}^{l}=\beta_{n}^{l}, l=1 \ldots 4
$$

where, $\beta_{n}^{l}=\sum_{p=1}^{4} s_{p}^{l} \tau_{n}^{p}\left(-\lambda_{p}^{2}\right)$, the definition of $s_{p}^{l}, \tau_{n}^{p}$, and $\lambda_{p}$ are show in Appendix B.

\section{References}

[1] K.H. Low, Comparisons of experimental and numerical frequencies for classical beams carrying a mass in-span, International Journal of Mechanical Sciences 41 (1999), 1515-1531.

[2] T.P. Chang, F.I. Chang and M.F. Liu, On the eigenvalues of a viscously damped simple beam carrying point mass and spring, Journal of Sound and Vibration 240(4) (2001), 769-778.

[3] H.Y. Lin and Y.C. Tsai, Free vibration analysis of a uniform multi-span beam carrying multiple spring-mass systems, Journal of Sound and Vibration 302 (2007), 442-456.

[4] W.Q. Chen, C.F. Lv and Z.G. Bian, Amixed method for bending and free vibration of beams resting on a Pasternak elastic foundation, Applied Mathematical Modelling 28 (2004), 877-890.

[5] M. Murgoze, On the alternative formulations of the frequency equation of a Bernoulli-Euler beam to which several spring-mass systems are attached in-span, Journal of Sound and Vibration 217 (1998), 585-595.

[6] J.S. Wu and T.F. Hsu, Free vibration analyses of simply supported beams carrying multiple point masses and spring-mass systems with mass of each helical spring considered, International Journal of Mechanical Sciences 49 (2007), 834-852.

[7] M. Murgoze and H. Batan, On the effect of an attached spring mass system on the frequency spectrum of a cantilevered beam, Journal of Sound and Vibration 195(1) (1996), 163-168.

[8] D. Zhou and T.J. Ji, Dynamic characteristics of a beam and distributed spring-mass system, International Journal of Solids and Structures 43 (2006), 5555-5569.

[9] K. Alsaif and M.A. Foda, Vibration suppression of a beam structure by intermediate masses and springs, Journal of Sound and Vibration 256(4) (2002), 629-645.

[10] Ö. Turhan, On the fundamental frequency of beams carrying a point mass: Rayleigh approximations versus exact solution, Journal of Sound and Vibration 230(2) (2000), 449-459.

[11] K.H. Low, On the eigenfrequencies for mass loaded beam under classical boundary condition, Journal of Sound and Vibration 215(2) (1998), 381-389. 
[12] K.H. Low, On the methods to derive frequency equations of beams carrying multiple masses, International Journal of Mechanical Sciences 43 (2001), 871-881.

[13] H.M. El-Khatib, B.R. Mace and M.J. Brennan, Suppression of bending waves in a beam using a tuned vibration absorber, Journal of Sound and Vibration 288 (2005), 1157-1175.

[14] M.A. De Rosa and M.J. Maurizi, The influence of concentrated masses and Pasternak soil on the free vibrations of beam-exact solution, Journal of Sound and Vibration 212(4) (1998), 573-581.

[15] J.S. Wu and H.M. Chou, Free vibration analysis of a cantilever beam carrying any number if elastically mounted point masses with the analytical and numerical combined method, Journal of Sound and Vibration 213(2) (1998), 317-332.

[16] D. Zhou, The vibrations of a cantilever beam carrying a heavy tip mass with elastic supports, Journal of Sound and Vibration 206(2) (1997), 275-279.

[17] C.H. Chang, Free vibration of a simply support beam carrying a rigid mass at the middle, Journal of Sound and Vibration 237(4) (2000), 733-744.

[18] P.D. Cha, Free vibrations of uniform timoshenko beams with lumped attachments, Journal of Sound and Vibration 211(2) (1998), $273-276$.

[19] B. Posiadala, Free vibrations of uniform timoshenko beams with attachments, Journal of Sound and Vibration 204(2) (1997), 359-369.

[20] W.L. Li and M. Daneils, A fourier series method for the vibrations of elastically restrained plates loaded with springs and masses, Journal of Sound and Vibration 252(4) (2002), 768-781.

[21] W.L. Li, X. Zhang, J. Du and Z. Liu, An exact series solution for the transverse vibration of rectangular plates with general elastic boundary supports, Journal of Sound and Vibration 321 (2009), 254-269.

[22] M. Berhann, The polynomial Eigenvalue Problem. PhD, thesis, University of Manchester, England, 2005.

[23] T. Ji, Understanding the interactions between people and structures, The Structure Engineers 81 (2003), 12-13.

[24] Q. Mao and S. Pietrzko, Free vibration analysis of stepped beams by using Adomian decomposition method, Applied Mathematics and Computation 217 (2010), 3429-3441. 

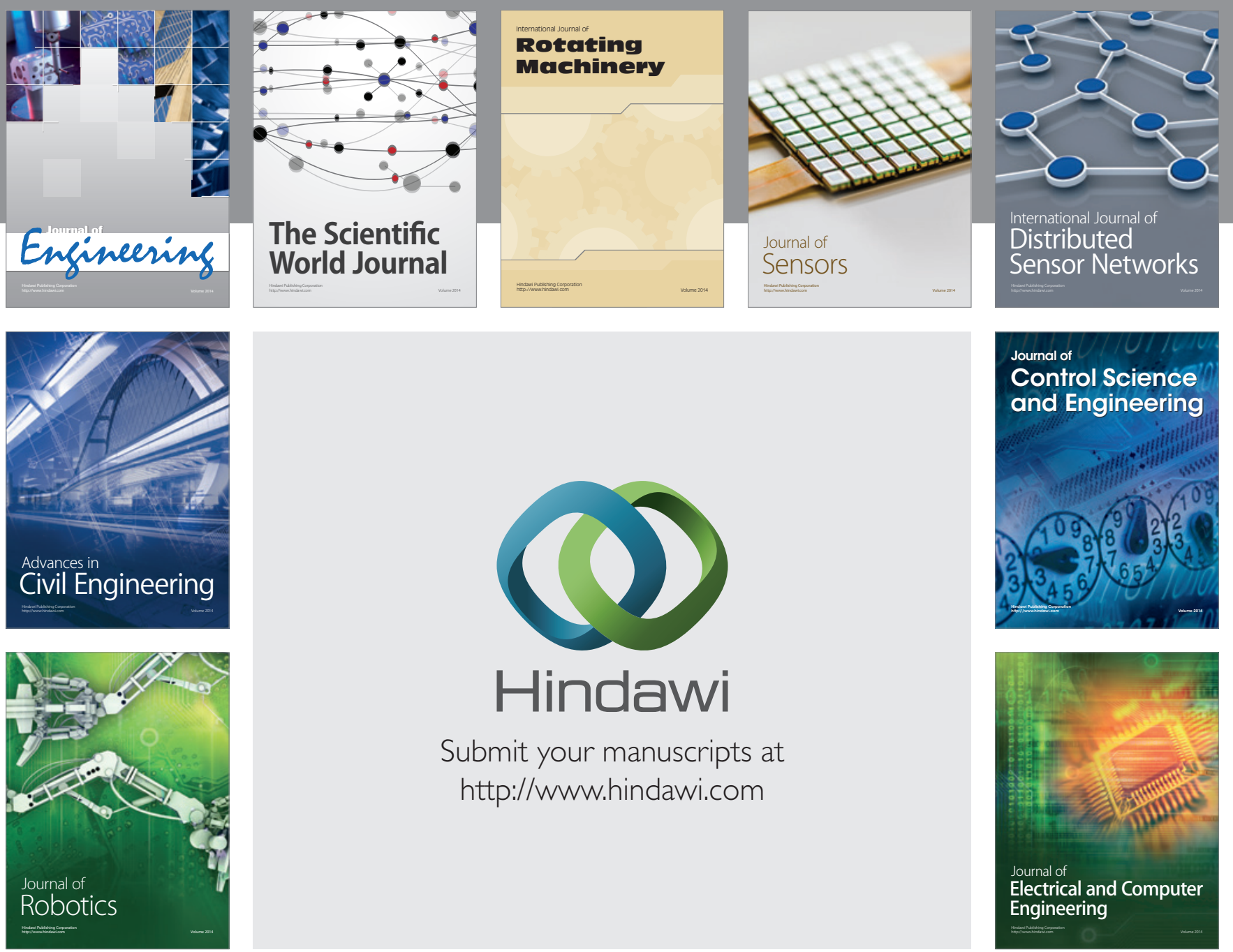

Submit your manuscripts at

http://www.hindawi.com
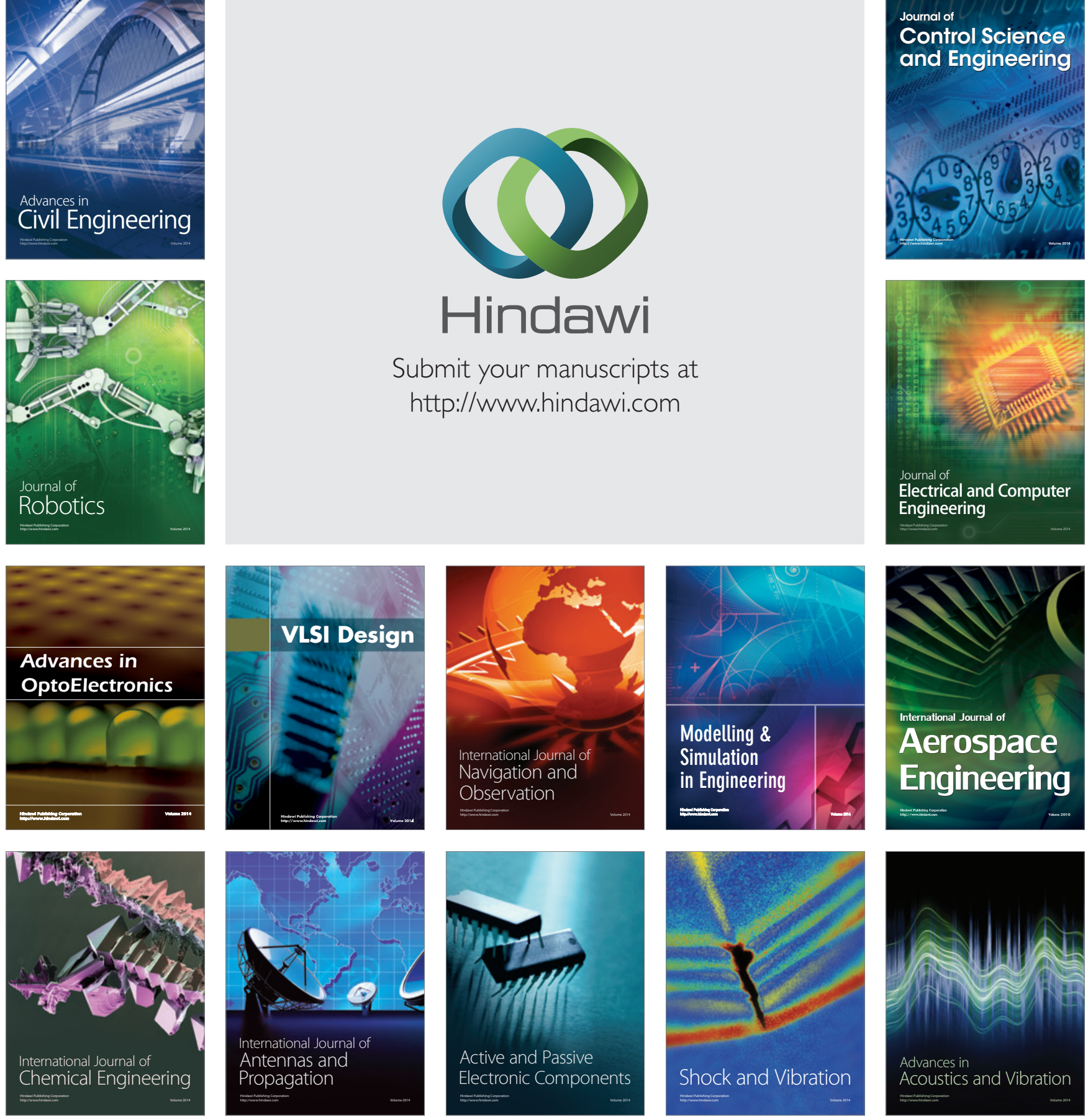